\title{
CEPHALOPHARYNGEAL SKELETONS OF Chrysomya megacephala (Fabricius, 1794) (DIPTERA: CALLIPHORIDAE) THIRD INSTAR LARVAE DISPLAYED RESISTANCE TO HOT-WATER KILLING METHOD - IMPLICATIONS IN FORENSIC ENTOMOLOGY PRACTICE
}

\author{
SHARIFAH SHAKILAH ABDULLAH ${ }^{1}$ and RAJA M. ZUHA ${ }^{1,2^{*}}$ \\ ${ }^{1}$ Forensic Science Programme, Faculty of Health Sciences, \\ (@) Basement 1, Tun Seri Lanang Library, \\ 43600 UKM Bangi, Selangor, Malaysia \\ ${ }^{2}$ Centre for Insect Systematics, Faculty of Science \& Technology, \\ 43600 UKM Bangi, Selangor, Malaysia \\ *E-mail: rmzuha@ukm.edu.my
}

Accepted 11 July 2020, Published online 31 December 2020

Forensic entomology is one of the most critical fields in death investigations with the main role to provide minimum post-mortem interval (mPMI) estimation based on insect specimens collected from decomposing human corpses and animal carcasses. During active decomposition stage, necrophagous insects that feed on decaying human or animal tissues can be used as the main reference to estimate mPMI (Greenberg \& Kunich, 2002). The calculation of $\mathrm{mPMI}$ is usually derived from the age of these insects which are predominantly dipterous larvae.

To preserve dipterous larvae for mPMI assessment, larvae must first be 'fixed' or killed with hot or boiling water $\left(60-100^{\circ} \mathrm{C}\right)$ (Lord \& Burger, 1983; Smith, 1986; Byrd, 2001; Adams \& Hall, 2003), before preservation in range of $75-90 \%$ ethanol (Amendt et al., 2007). In this preliminary step, killing larvae with hot water could halt the developmental size, clean, and expand their sizes to maximum length (Rotheray, 2010). Hence, this technique can be considered mandatory because direct preservation of larvae in ethanol could cause shrinkage, and worst, putrefy and darken the larvae (Amendt et al., 2007; Rosilawati et al., 2014). This will subsequently compromise its evidential values by greatly underestimating $\mathrm{mPMI}$ and making species identification more difficult.

However, killing larvae with hot water at the death scene could sometimes be impractical. The current guideline by Amendt et al. (2007) recommends that larval killing should be undertaken

\footnotetext{
* To whom correspondence should be addressed.
}

as soon as possible in the laboratory, but it would be impossible if death investigations occurred in remote locations from the lab. To address this limitation, few experiments have been employed to compare the effects of killing methods and preservation techniques on larval specimens, particularly on the differences between hot water kill or direct immersion of larvae in preservatives. Previous findings indicated larval body length was affected by both techniques, i.e. the larvae expanded when killed with hot water and shortened when immersed directly in preservatives. Tantawi and Greenberg (1993) recorded up to 9.7 hours of underage error due to larval shrinkage of Protophorma terranovae (Robineau-Desvoidy, 1830) and up to $19.2 \mathrm{hr}$ in Calliphora vicina (Robineau-Desvoidy 1830) when directly preserved in $70 \%$ ethanol. Larval shrinkages were also reported in Calliphora vomitoria (Linnaeus, 1758) and Lucilia sericata (Meigen, 1826) when directly preserved in $80 \%$ ethanol instead of $70 \%$ ethanol (Adams \& Hall, 2003). Other than the effect of killing techniques on larval body length, storage periods, ethanol concentrations and other solution substitutes such as Kahle's and 10\% formalin also produced an inconsistent impact on larval body length (Day \& Wallman, 2008; Richards et al., 2013; Rosilawati et al., 2014).

As a result, other larval growth parameters have been proposed to age larva for mPMI assessment. This includes larval width (Day \& Wallman, 2006), weight (Wells \& LaMotte, 1995), and even the size of the larval guts (Amendt et al., 2007; Tantawi \& Greenberg, 1993). Modern and non-invasive 
approaches to enhance the accuracy of larval size measurements include digital image analysis (Bourne et al., 2019) and the application of geometric micrometer (Bugelli et al., 2017). Hence, larval mouthparts, or the cephalopharyngeal skeleton have been suggested as an alternative parameter to larval body length because it is more rigid than the larval body (Rabbani \& Zuha, 2007).

In cyclorrhaphan Diptera, the cephalopharyngeal skeleton is the larval mouthparts that invaginate in the cephalic region. It is divided into three segments i.e. tentoropharyngeal sclerite (basal/pharyngeal sclerite), hypopharyngeal sclerite (intermediate sclerite), and mandibles (mouth hooks) (Teskey, 1981). The largest section of the cephalopharyngeal skeleton, the pharyngeal sclerite, has four projections i.e. two dorsal and two ventral cornua and joined anteriorly by a dorsal bridge. The composition of these structures has been configured as morphometric landmarks that can be utilized to measure the growth of forensically important Calliphoridae larva (Chaiwat et al., 2012; Nateeworanart et al., 2010). Rabbani and Zuha (2016) subsequently studied the growth of Hypopygiopsis violacea (Macquart, 1835) based on cephalopharyngeal skeleton morphometry and discovered consistent growth measurement. They also found that cephalopharyngeal skeleton size unaffected by preservatives when stored up to seven days.

Studying the efficacy of the cephalopharyngeal skeleton as a growth parameter is therefore necessary as in the later studies recorded it grew proportionally with larval body size during active feeding stage (Eliza \& Zuha, 2018; Sim \& Zuha, 2019). The current study compared the effect of hot water and direct killing by immersion in $70 \%$ ethanol on the cephalopharyngeal skeleton and total body size of third instar Chrysomya megacephala (Fabricius, 1786) larvae, a forensically important blowfly in Malaysia, Thailand, and the rest of the world (Lee et al., 2004; Sukontason et al., 2008; Thevan et al., 2010; Badenhorst \& Villet, 2018). In local natural surroundings, C. megacephala has been observed to be among the earliest to arrive to feed and oviposit on the decaying organic materials especially human corpses both indoors and outdoors (Lee et al., 2004). We hypothesized that the cephalopharyngeal skeleton length of the third instar C. megacephala larvae and its total body length react differently when killed with hot water or directly preserved in $70 \%$ ethanol for seven days. The information gained from this study would further support the premise of using the cephalopharyngeal skeleton as an alternative growth parameter to larval body length.
This study was conducted in three repetitions from 14 August 2018 to 28 August 2018 (study replicate 1) and 11 October 2018 to 28 October 2018 (study replicate 2 and 3, ran concurrently but in separate environmental conditions). A baited trap, consisting of approximately $400 \mathrm{~g}$ raw fish - yellow stripe scads, Selaroides leptolepis Cuvier, 1833 and Indian Mackerel, Rastrelliger kanagurta (Cuvier, 1817), and $400 \mathrm{~g}$ raw cow's liver were placed inside a black plastic container. They were left exposed for $8 \mathrm{hr}$ during the daytime from 0900 to $1700 \mathrm{hr}$ in an open area adjacent to the Forensic Entomology Laboratory, Forensic Science Programme, Faculty of Health Sciences, Universiti Kebangsaan Malaysia, Bangi. A single batch of calliphorid eggs presumably C. megacephala was carefully collected by using fine-tip forceps and transferred into a $700 \mathrm{~mL}$ plastic rearing container with $70 \mathrm{~g}$ fresh cow's liver on $2 \mathrm{~cm}$ sawdust layered at the bottom. Rearing took place at an ambient temperature of $22.0-34.0^{\circ} \mathrm{C}$ (study replicate 1 ) and $25.2-28.5^{\circ} \mathrm{C}$ (study replicate 2 and 3), and relative humidity (RH) of $60.5-87.5 \%$ (study replicate 1) and $74.5-97.5 \%$ (study replicate 2 and 3). These data were acquired from a data logger (Lascar, UK) placed next to the rearing container.

On the next day at $0900 \mathrm{hr}$, the first instar larvae that newly emerged were transferred evenly into two separate rearing containers representing hot water kill (HWK) and direct kill (DK) in 70\% ethanol. To ensure sample homogeneity, each rearing container used in this study were similar, consisting of 20 first instar larvae each and supplied with $70 \mathrm{~g}$ fresh cow's liver obtained from the same source. The remaining larvae that were not used for sampling were kept in a separate container and reared in similar conditions until the adult stage to facilitate species identification. After $48 \mathrm{hr}$, all 20 larvae from HWK container that was by then already in the third instar phase (based on the three slits on the posterior spiracles) were killed by immersion in hot water $\left(80^{\circ} \mathrm{C}\right)$ for $60 \mathrm{sec}$ (Adams \& Hall, 2003; Amendt et al., 2007) and subsequently transferred to universal glass vials containing $20 \mathrm{~mL} 70 \%$ ethanol. To ensure the accuracy of the temperature, Fluke 51 II handheld digital probe thermometer with type $\mathrm{K}$ thermocouple was immersed in $1.5 \mathrm{~L}$ boiling water. Another 20 third instar larvae from DK container were directly transferred universal glass vials containing $20 \mathrm{~mL} 70 \%$ ethanol. The type of vials and amount of preservatives used in this study were decided based on forensic specimens received by the Forensic Entomology Laboratory, UKM. All larvae in HWK and DK vials were preserved at room temperature and relative humidity for seven days. 
Based on the data logger (Lascar, UK) placed in the proximity of the vials, the room temperatures were recorded as $20.5-24.5^{\circ} \mathrm{C}$ (study replicate 1 ) and $21.0-24.5^{\circ} \mathrm{C}$ (study replicate 2 and 3 ), and $\mathrm{RH}$ were $58.5-88.5 \%$ (study replicate 1 ) and $67.5-90.0 \%$ (study replicate 2 and 3 ). In this study, the larval lengths before HWK and DK were not measured.

After seven days of storage, all larvae from HWK and DK were withdrawn from respective vials and each larval body length was measured based on lateral body segments i.e. furthest part of the head and the last abdominal segment (Day \& Wallman, 2008). After measuring larval body length, the cephalopharyngeal skeleton was removed from the larva and immersed in $10 \% \mathrm{KOH}$ for $15 \mathrm{~min}$. The internal tissues surrounding the cephalopharyngeal skeleton were carefully removed in $10 \% \mathrm{KOH}$ and subsequently transferred into $10 \%$ acetic acid for 10 min. Cephalopharyngeal skeleton was then soaked in $70 \%$ ethanol for $20 \mathrm{~min}$ before they were mounted onto a glass slide in lateral position using Berlese fluid and covered by a $6 \mathrm{~mm}$ round coverslip (Sim \& Zuha, 2019). Measurements of the cephalopharyngeal skeleton were conducted immediately after the mounting process based on inter landmark distances between anterodorsal process (tip of the dorsal bridge) to dorsal cornu (ADP-DC), the anterodorsal process to ventral cornu (ADP-VC), and dorsal cornu to ventral cornu (DC-VC) (Nateeworanart et al., 2010; Nuñez \& Liria, 2016; Rabbani \& Zuha, 2017) (Figure 1). Larval body length and cephalopharyngeal skeleton length were measured using Nikon SMZ745T stereomicroscope fitted with Toupcam 12megapixel industrial digital camera. The system used for measurement was Toupview software version 3.7, with a measurement accuracy of length at $\pm 0.001 \mathrm{~mm}$ based on calibration at each magnification step using a stage micrometer on the highest resolution setting $(4000 \times 3000$ pixels $)$. Descriptive and inferential statistics were conducted on the morphometric data of larval body length and cephalopharyngeal skeleton length based on independent groups i.e. HWK and DK. Within-group sample variance $\left(s^{2}\right)$ were calculated to evaluate individual data consistencies in larval body length and cephalopharyngeal skeleton. To test mean differences, we performed an independent sample $t$-test on the normally distributed samples $(a=0.05)$ in SPSS 20 separately in each study replicate. Repeated measure analysis such as paired sample $t$-test was not used because samples were not measured before and after treatment. Instead, they were only compared after treatment. Before analysis, an inspection of Shapiro-Wilks and Levene's tests across all study replicates indicated that the assumption of normality $(p>0.05)$ and homogeneity of variances was not violated $(p>0.05)$. Species identification was conducted based on remaining third instar larvae and adults which were reared from calliphorid eggs. Species confirmation was based on taxonomic keys in Kurahashi et al. (1997) for adults and Greenberg and Kunich (2002) for larvae. All specimens that were used as experimental species in this study were confirmed as C. megacephala.

Morphometry of $C$. megacephala larval body length and cephalopharyngeal skeleton length in HWK and DK across three study replicates are presented in Table 1. Across all study replicates, cephalopharyngeal skeletons size, represented by inter-landmark distances ADP-DC, ADP-VC and $\mathrm{DC}-\mathrm{VC}$, was neither affected when preserved in

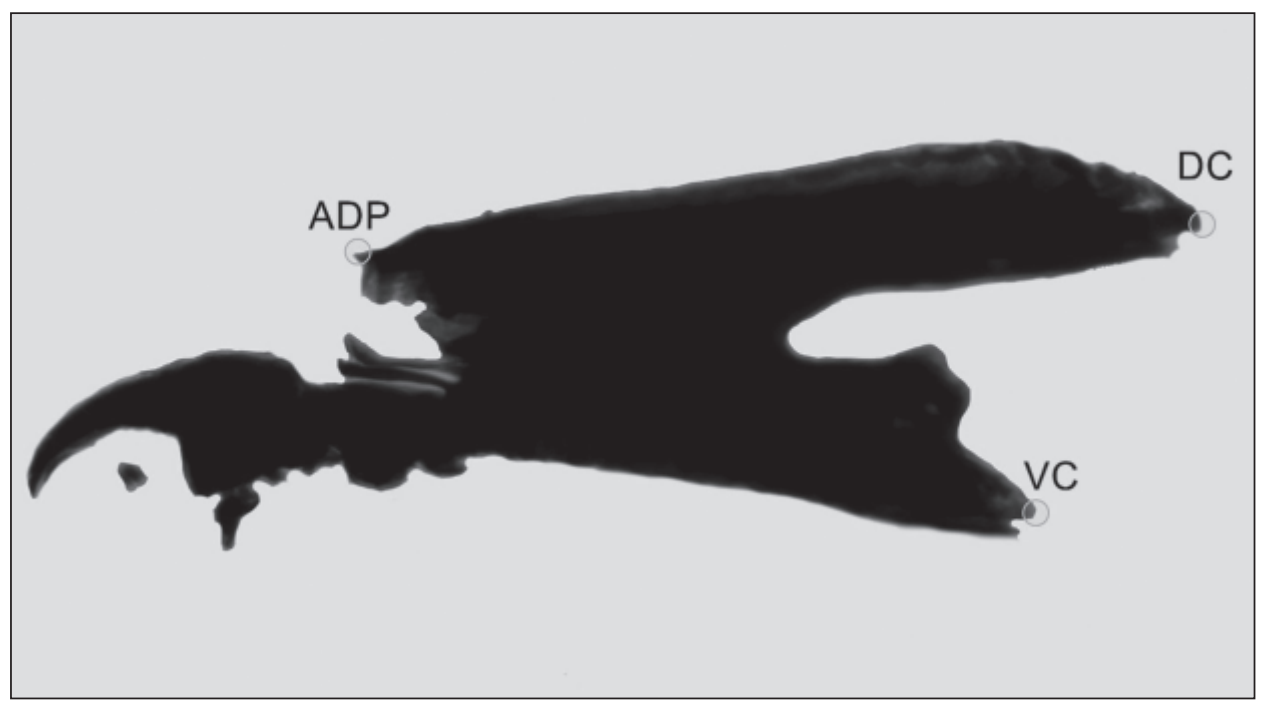

Fig. 1. Cephalopharyngeal skeleton size represented by interlandmark distances of anterodorsal process-dorsal cornu (ADP-DC), anterodorsal process-ventral cornu (ADP-VC), and dorsal cornuventral cornu (DC-VC). 
Table 1. Descriptive summary of larval body length (BL) and cephalopharyngeal skeleton length by hot water killed (HWK) and direct killed (DK) for all study replicates. Cephalopharyngeal skeleton length is represented by interlandmark distances of anterodorsal process-dorsal cornu (ADP-DC), anterodorsal process-ventral cornu (ADP-VC), and dorsal cornu-ventral cornu (DC-VC).

\begin{tabular}{|c|c|c|c|c|c|c|c|c|c|c|c|c|c|c|}
\hline \multirow{2}{*}{ Rep } & \multirow{2}{*}{ Parameter } & \multirow{2}{*}{$N$} & \multicolumn{2}{|c|}{ Mean (M) } & \multicolumn{2}{|c|}{ Min } & \multicolumn{2}{|c|}{ Max } & \multicolumn{2}{|c|}{ Range } & \multicolumn{2}{|c|}{$\mathrm{s}$} & \multicolumn{2}{|c|}{$s^{2}$} \\
\hline & & & HWK & DK & HWK & DK & HWK & DK & HWK & DK & HWK & DK & HWK & DK \\
\hline \multirow{4}{*}{1} & $\mathrm{BL}$ & 20 & 16.190 & 15.039 & 15.243 & 13.466 & 17.555 & 16.28 & 2.312 & 2.752 & 0.609 & 0.749 & 0.371 & 0.561 \\
\hline & ADP-DC & 20 & 1.103 & 1.120 & 0.960 & 0.993 & 1.273 & 1.204 & 0.313 & 0.211 & 0.082 & 0.055 & 0.007 & 0.003 \\
\hline & ADP-VC & 20 & 0.895 & 0.906 & 0.775 & 0.820 & 1.060 & 1.002 & 0.285 & 0.182 & 0.070 & 0.052 & 0.005 & 0.003 \\
\hline & DC-VC & 20 & 0.446 & 0.429 & 0.351 & 0.384 & 0.499 & 0.491 & 0.148 & 0.107 & 0.033 & 0.029 & 0.001 & 0.001 \\
\hline \multirow{4}{*}{2} & $\mathrm{BL}$ & 20 & 15.977 & 13.538 & 15.014 & 12.270 & 17.406 & 14.709 & 2.392 & 2.439 & 0.597 & 0.577 & 0.356 & 0.333 \\
\hline & ADP-DC & 20 & 1.243 & 1.237 & 1.178 & 1.115 & 1.332 & 1.298 & 0.154 & 0.183 & 0.047 & 0.045 & 0.002 & 0.002 \\
\hline & ADP-VC & 20 & 1.085 & 1.056 & 1.013 & 0.967 & 1.207 & 1.197 & 0.194 & 0.23 & 0.058 & 0.063 & 0.003 & 0.004 \\
\hline & DC-VC & 20 & 0.549 & 0.552 & 0.489 & 0.495 & 0.633 & 0.636 & 0.144 & 0.141 & 0.038 & 0.039 & 0.001 & 0.002 \\
\hline \multirow{4}{*}{3} & $\mathrm{BL}$ & 20 & 14.36 & 12.695 & 12.668 & 11.009 & 15.731 & 13.638 & 3.068 & 2.629 & 0.737 & 0.687 & 0.543 & 0.471 \\
\hline & ADP-DC & 20 & 1.255 & 1.26 & 1.183 & 1.186 & 1.297 & 1.338 & 0.114 & 0.152 & 0.034 & 0.041 & 0.001 & 0.002 \\
\hline & ADP-VC & 20 & 1.064 & 1.084 & 0.984 & 0.963 & 1.157 & 1.179 & 0.173 & 0.216 & 0.048 & 0.057 & 0.002 & 0.003 \\
\hline & DC-VC & 20 & 0.523 & 0.520 & 0.447 & 0.463 & 0.585 & 0.605 & 0.138 & 0.142 & 0.036 & 0.043 & 0.001 & 0.002 \\
\hline
\end{tabular}

Table 2. Mean length comparisons of larval body length $(B L)$ and cephalopharyngeal skeleton length using independent sample $t$-test $(a=0.05)$ by hot water killed $(H W K)$ and direct killed (DK). Cephalopharyngeal skeleton length is represented by interlandmark distances of anterodorsal process-dorsal cornu (ADP-DC), anterodorsal process-ventral cornu (ADP$\mathrm{VC})$, and dorsal cornu-ventral cornu (DC-VC)

\begin{tabular}{|c|c|c|c|c|c|c|}
\hline \multirow{2}{*}{$\begin{array}{c}\text { Study } \\
\text { Replicate }\end{array}$} & \multirow{2}{*}{ Parameter } & \multirow{2}{*}{ Mean Difference } & \multicolumn{2}{|c|}{ Standard Error of Mean } & \multirow{2}{*}{$p$-value } & \multirow{2}{*}{ Effect size (Cohen's a } \\
\hline & & & HWK & DK & & \\
\hline \multirow{4}{*}{1} & $B L$ & 1.150 & 0.136 & 0.167 & $<0.05$ & 1.685 \\
\hline & ADP-DC & -0.017 & 0.018 & 0.012 & 0.441 & 0.243 \\
\hline & ADP-VC & -0.011 & 0.016 & 0.012 & 0.592 & 0.178 \\
\hline & DC-VC & 0.017 & 0.007 & 0.006 & 0.084 & 0.557 \\
\hline \multirow{4}{*}{2} & $\mathrm{BL}$ & 2.440 & 0.134 & 0.129 & $<0.05$ & 4.154 \\
\hline & ADP-DC & 0.006 & 0.011 & 0.010 & 0.672 & 0.130 \\
\hline & ADP-VC & 0.029 & 0.013 & 0.014 & 0.135 & 0.480 \\
\hline & DC-VC & -0.003 & 0.009 & 0.009 & 0.792 & 0.078 \\
\hline \multirow{4}{*}{3} & $\mathrm{BL}$ & 1.666 & 0.165 & 0.154 & $<0.05$ & 2.338 \\
\hline & ADP-DC & -0.005 & 0.007 & 0.009 & 0.684 & 0.133 \\
\hline & ADP-VC & -0.020 & 0.010 & 0.012 & 0.242 & 0.361 \\
\hline & DC-VC & 0.004 & 0.008 & 0.010 & 0.765 & 0.101 \\
\hline
\end{tabular}

HWK nor DK after seven days. In contrast to larval body length, significant changes were detected between those preserved in HWK and DK (Table 2). Larval body lengths in HWK were found to be significantly different from those in DK in all study replicates $(p<0.05)$. In study replicate 1 , larval body length in HWK were $1.150 \mathrm{~mm}$ longer, 95\% CI $(0.713,1.587)$, than those in DK, $t(38)=5.329$, $p<0.05, d=1.685$ whilst in study replicate 2 larvae in HWK were $2.240 \mathrm{~mm}$ longer, 95\% CI (2.064, $2.815)$, than the larvae in DK, $t(38)=13.136, p<0.05$, $d=4.154$. Similar pattern was also observed in study replicate 3, whereby larvae in HWK were $1.666 \mathrm{~mm}$ longer, 95\% CI $(1.210,2.122)$, than those in DK, $t(38)=7.392, p<0.05, d=2.338$. In contrast, significant test showed no difference between HWK and DK groups for cephalopharyngeal skeleton (ADP-DC, ADP-VC and DC-VC). In replicate 1, ADP-DC 
$t(38)=-0.778, p=0.441, d=0.243$, ADP-VC $t(38)=$ $-0.540, p=0.592, d=0.178$ and DC-VC $t(38)=1.775$, $p=0.084, d=0.557$. In replicate 2 , ADP-DC $t(38)=$ $0.427, p=0.672, d=0.130$, ADP-VC $t(38)=1.526$, $p=0.135, d=0.480$ and $\mathrm{DC}-\mathrm{VC} t(38)=-0.266$, $p=0.792, d=0.557$ whilst in replicate 3 , ADP-DC $t(38)=-0.410, p=0.684, d=0.133$, ADP-VC $t(38)=$ $-1.188, p=0.242, d=0.361$ and DC-VC $t(38)=0.302$, $p=0.765, d=0.101$.

After seven days, C. megacephala third instar larvae that were killed with hot water before preservation in $70 \%$ ethanol were longer than those directly preserved in $70 \%$ ethanol. The differences were statistically significant with mean differences ranged from 1.15 to $2.44 \mathrm{~mm}$. Previous studies using HWK before preservation in $70 \%$ ethanol also recorded an expansion of larval size at varying degrees (Adams \& Hall, 2003). Specifically, in the case of $C$. megacephala, the size of the third instar larvae recorded an average length increase from $11.98 \pm 2.82$ to $12.17 \pm 2.86 \mathrm{~mm}$ when killed with $80^{\circ} \mathrm{C}$ hot water before preservation in $70 \%$ ethanol (Rosilawati et al., 2014). On other species, Adams \& Hall (2003) experimented with the effect of HWK followed by preservation in $80 \%$ ethanol on Ca. vomitoria and L. sericata larvae, with the size expansions, were greater than those directly killed in $80 \%$ ethanol. Furthermore, Adams \& Hall (2003) provided evidence that immersion of larvae longer in boiling water produced a better quality of larval preservation. To minimize the effect of killing methods and preservatives on larval length, Bugelli et al. (2017) recommended, among others, to perform measurement right after killing and use highly concentrated alcohol with a conducive storing environment.

In contrast, cephalopharyngeal skeleton size, represented by inter landmark distances of ADP-DC, ADP-VC, and DC-VC, did not exhibit any significant changes when either killed with hot water or directly preserved in $70 \%$ ethanol for seven days. Because of the firm characteristic of the cephalopharyngeal skeleton, both killing methods did not affect the size. Most importantly, results from the current study suggesting that cephalopharyngeal skeletons can be used as an alternative growth parameter to estimate larval age after larval body length was found to be affected by various practical factors. For example, Sim \& Zuha (2019) showed that the cephalopharyngeal skeleton size of $C$. megacephala had a linear growth pattern during the active feeding period. Likewise, in other calliphorid species, the cephalopharyngeal skeleton could show positive trajectories throughout larval development as observed in $H$. violacea (Rabbani \& Zuha, 2017) and Hemipyrellia ligurriens (Wiedemann, 1830) (Eliza \& Zuha, 2018).
On that account, the usability of the cephalopharyngeal skeleton as an alternative growth parameter warranted further investigations to address limitations and other factors that could affect both larval and cephalopharyngeal skeleton morphometries from the current study. First, current research only showed morphometric comparisons between larvae after preservation of seven days in $70 \%$ ethanol through HWK and DK methods. The main limitation was that the size of larvae before killing in hot water or direct preservation in $70 \%$ ethanol was not obtained and compared with after seven days of preservation. We recommend the future study to prepare sample replicates of larvae to represent the larval body and cephalopharyngeal skeleton size before preservation (day 0 ) or to use a non-destructive method to measure live larvae as suggested by Bourne et al. (2019). Secondly, due to ontogenetic allometry of the cephalopharyngeal skeleton that develops in linear form as its total body size (Eliza \& Zuha, 2018; Sim \& Zuha, 2019), the effect of killing techniques on a range of larval age groups or instars could also be different. The first instar larva is more delicate than the third instar larva and therefore, its larval body structure including cephalopharyngeal skeleton could be more susceptible to the killing method. As reported by Tantawi \& Greenberg (2003), direct preservation in $70 \%$ ethanol causing more shrinkage in young third instar larvae than the older ones. Thirdly, the effects of preservation techniques could be speciesspecific as dipterous larval morphology is greatly diverse (Ferrar, 1987) and the reactions from ethanol tonicity could be different across species. We recommend future experiment to compare the effects of different ethanol concentrations such as 70 to $95 \%$ as recommended by Amendt et al. (2007), on both larval body and cephalopharyngeal skeleton. Duration of immersion could also be variable instead of only observing the effect after seven days as in the current study. Lastly, the cephalopharyngeal skeleton is a three-dimensional shape but its length was configured based on a two-dimensional image. Therefore, planarity could be the main issue that affects inter-landmark distances measurement as a landmark position could be influenced by structure depth and image quality. We minimized the effect by using images taken from a similar plane at fixed focal length and lighting (Sim \& Zuha, 2019). However, for future study, using geometrical landmarks to obtain centroid size as previously applied in previous studies could provide a better estimation of cephalopharyngeal skeleton size (Pélabon et al., 2013; Nuñez \& Liria, 2016; Sim \& Zuha, 2019). Centroid size is the square root of the sum of squared distances of the landmarks from the centroid, i.e. the calculated 'center of gravity', and 
has been widely used in the geometric morphometric analysis to measure the size of organismal forms (Zelditch et al., 2004).

Cephalopharyngeal skeleton of the third instar C. megacephala can potentially be a reliable growth parameter to age larvae for mPMI assessment, particularly when larval killing with hot water is not feasible at death locations. Killing with hot water showed no significant effect on cephalopharyngeal skeleton size after being stored for seven days. With previous records showed cephalopharyngeal skeleton size positively correlated with larval body size, it is imperative to establish proper larval developmental models based on cephalopharyngeal skeleton dimensions so that it can be used as a reference for forensic entomologists to estimate larval age. Further reliability and validity tests are also required to address practical issues about the feasibility of the cephalopharyngeal skeleton before being implemented as part of recommended guidelines for mPMI analysis. This includes establishing a standard protocol to process cephalopharyngeal skeleton and the configuration of morphometric landmarks.

\section{ACKNOWLEDGEMENT}

The authors would like to thank the laboratory staff from Forensic Science Program, Faculty of Health Sciences, UKM, particularly Miss Atiah Ayunni Abdul Ghani and Ms. Balkhis Bashuri, for providing the equipment and chemicals required. We also wish to thank Miss Sim Li Xuan for her kind assistance during the research.

\section{REFERENCE}

Adams, Z.J.O. \& Hall, M.J.R. 2003. Methods used for the killing and preservation of blowfly larvae, and their effect on post-mortem larval length. Forensic Science International, 138: 50-61.

Amendt, J., Campobasso, C.P., Gaudry, E., Reiter, C., LeBlanc, H.N. \& Hall, M.J.R. 2007. Best practice in forensic entomology-standards and guidelines. International Journal of Legal Medicine, 121(2): 90-104.

Badenhorst, R. \& Villet, M.H. 2018. The uses of Chrysomya megacephala (Fabricius, 1794) (Diptera: Calliphoridae) in forensic entomology. Forensic Sciences Research, 3(1): 2-15.
Bourne, D.R., Kyle, C.J., LeBlanc, H.N. \& Beresford, D. 2019. A rapid, non-invasive method for measuring live or preserved insect specimens using digital image analysis. Forensic Science International: Synergy, 1: 140-145.

Bugelli, V., Campobasso, C.P., Verhoff, M.A. \& Amendt, J. 2017. Effects of different storage and measuring methods on larval length values for the blow flies (Diptera: Calliphoridae) Lucilia sericata and Calliphora vicina. Science \& Justice, 57: 159-164.

Byrd, J.H. 2001. Laboratory rearing of forensic insects. In: Forensic Entomology: The Utility of Arthropods in Legal Investigations. J.H. Byrd and J.L. Castner (Eds). CRC Press, Boca Raton. pp. 121-142.

Chaiwat, C., Nateeworanart, S. \& Jiraviriyakul, A. 2012. Length of cephalopharyngeal skeleton of Chrysomya rufifacies, third instar larvae, collected from Naresuan University, Phitsanulok Province. Forensic Medicine Journal 4: 127-134.

Day, D.M. \& Wallman, J.F. 2006. Width as an alternative measurement to length for postmortem interval estimations using Calliphora augur (Diptera: Calliphoridae) larvae. Forensic Science International 159: 158-167.

Day, D.M. \& Wallman, J.F. 2008. Effect of preservative solutions on preservation of Calliphora augur and Lucilia cuprina larvae (Diptera: Calliphoridae) with implications for post-mortem interval estimates. Forensic Science International, 179(1): 1-10.

Eliza, P. \& Zuha, R.M. 2018 Preliminary assessment of cephalopharyngeal skeleton length and body length of Hemipyrellia ligurriens (Wiedermann) (Diptera: Calliphoridae) larvae as potential parameters to estimate minimum post mortem interval. Egyptian Journal of Forensic Sciences, 8: 39.

Ferrar, P. 1987. A guide to the breeding habits and immature stages of Diptera Cyclorrhapha (Part I). In: Entomonograph volume 8- 1987. Lyneborg, L. (Ed.) Scandinavian Science Press Ltd, Vinderup. pp. 488.

Greenberg, B. \& Kunich, J.C. 2002. Entomology and The Law: Flies as Forensic Indicators. Cambridge University Press, Cambridge.

Kurahashi, H., Benjaphong, N. \& Omar, B. 1997. Blow flies (Insecta: Diptera: Calliphoridae) of Malaysia and Singapore. Raffles Bulletin of Zoology, Supp No. 5: 1-88. 
Lee, H.L., Krishnasamy, M., Abdullah, A.G. \& Jeffery, J. 2004 Review of forensically important entomological specimens in the period of 19722002. Tropical Biomedicine, 21(2): 69-75.

Lord, D.L. \& Burger, J.F. 1983. Collection and preservation of forensically important entomological materials. Journal of Forensic Sciences, 28: 936-944.

Nateeworanart, S., Bunchu, N. \& Vitta, A. 2010. Cephalopharyngeal skeleton measurement of Chrysomya megacephala, third instar larvae, collected from Naresuan University, Phitsanuk Province. Journal of the Medical Technologist Association of Thailand, 38: 3100-3105.

Nuñez J. \& Liria, J. 2016. Cephalopharyngeal geometric morphometrics in three blowfly species (Diptera: Calliphoridae). Journal of Entomology and Zoology Studies 4(1): 338-341.

Pélabon, C., Bolstad, G.H. \& Egset, C.K. 2013. On the relationship between ontogenetic and static allometry. The American Naturalist, 181(2): 195-212.

Rabbani, A. \& Zuha, R.M. 2017. Cephalopharyngeal skeleton morphometry of Hypopygiopsis violacea (Macquart) (Diptera: Calliphoridae) A preliminary assessment for its application in forensic entomology. Journal of Entomology and Zoology Studies 5(1): 777-782.

Rotheray, G. 2010. Immature stages of Diptera. In: A Dipterist's Handbook. P.J. Chandler (Ed). The Amateur Entomologists' Society, Essex. pp. 133-144.

Richards, C.S., Rowlinson, C.C. \& Hall, M.J.R. 2013. Effects of storage temperature on the change in size of Calliphora vicina larvae during preservation in $80 \%$ ethanol. International Journal of Legal Medicine, 127(1): 231-241.

Rosilawati, R., Omar, B. \& Syamsa, R.A. 2014. Effects of preservatives and killing methods on morphological features of a forensic fly, Chrysomya megacephala (Fabricius, 1794) larva. Tropical Biomedicine, 31: 785-791.
Sim, L.X. \& Zuha, R.M. 2019. Chrysomya megacephala (Fabricius, 1794) (Diptera: Calliphoridae) development by landmark-based geometric morphometrics of cephalopharyngeal skeleton: a preliminary assessment for forensic entomology application. Egyptian Journal of Forensic Sciences, 9: 55.

Smith, K.G.V. 1986. A Manual of Forensic Entomology. British Museum (Natural History), London. 205 pp.

Sukontason, K., Piangjai, S., Siriwattanarungsee, S. \& Sukontason, K.L. 2008. Morphology and developmental rate of blow flies Chrysomya megacephala and Chrysomya rufifacies in Thailand: application in forensic entomology. Parasitology Research, 102(6): 1207-1206.

Tantawi, T.I. \& Greenberg, B. 1993. The effect of killing and preservative solutions on estimates of maggot age in forensic cases. Journal of Forensic Sciences, 38(3): 702-707.

Teskey, H.J. 1981. Morphology and terminology larvae. In: Manual of Nearctic Diptera, vol 1. J.F. McAlpine, B.V. Peterson, G.E. Shewell, H.J. Teskey, J.R. Vockeroth \& D.M. Wood (Eds.) Minister of Supply and Services Canada, Quebec. pp. 74-77.

Thevan, K., Ahmad, A.H., Md Rawi, C.S. \& Singh, B. 2010. Growth of Chrysomya megacephala (Fabricius) maggots in a morgue cooler. Journal of Forensic Sciences, 55: 1656-1658.

Wells, J.D. \& LaMotte, L.R. 1995. Estimating maggot age from weight using inverse prediction. Journal of Forensic Sciences, 40(4): 585-590.

Zelditch, M.L., Swiderski, D.L. \& David Sheets, H. 2012. Geometric Morphometrics For Biologists: A Primer. 2nd Ed. Academic Press, London. pp.59-60. 
